K. Ito

Nagoya Math. J.

Vol. 38 (1970), 181-183

\title{
THE TOPOLOGIGAL SUPPORT OF GAUSS MEASURE ON HILBERT SPACE
}

\author{
KIYOSI ITO \\ dedicated to Professor K. Ono for his sixtieth birthday
}

\section{Introduction}

Let $X$ be a Hilbert space. The topological support of a Radon probability measure $P$ on $X$ is the least closed subset $M$ of $X$ that carries the total measure 1. A closed subset $M$ of $X$ is called a linear subvariety if

$x, y \in M$ implies $x+(1-\alpha) y \in M$ for every $\alpha \in R^{1}$, or equivalently if $M=a+Y$ for some $a \in X$ and some closed linear subspace $Y$ of $X$. A Radon probability measure $P$ on $X$ is called a Gauss measure if for every $a \in X$, the image measure of $P$ by the map

$$
f_{a}(x)=(a, x): X \longrightarrow R^{1}
$$

is a Gauss measure on $R^{1}$.

The purpose of this note is to prove

Theorem. Let $P$ be a Gauss measure on a Hilbert space $X$. Then the topological support $S(P)$ of $P$ is a linear subvariety of $X$.

This fact is obvious in case $X$ is finite dimensional but we need a small trick to discuss the infinite dimensional case as we shall see below.

\section{Proof of the theorem.}

Since $P$ is a Gauss measure, its characteristic functional

$$
C(z)=\int_{X} e^{i(z, x)} P(d x)
$$

is expressed as

$$
C(z)=\exp \left\{i(z, m)-\frac{1}{2} \sum_{k} v_{k}\left(z, e_{k}\right)^{2}\right\}
$$

Received July 3, 1969 
where $\left\{e_{k}\right\}$ is an orthonormal sequence (finite or countable) and

$$
z \in X, \quad v_{k}>0 \quad \sum_{k} v_{k}<\infty .
$$

By the translation $x \longrightarrow x+m$, we can assume that $m=0$, namely that

$$
C(z)=\exp \left\{-\frac{1}{2} \sum_{k} v_{k}\left(z, e_{k}\right)^{2}\right\}
$$

Let $Y$ be the closed linear subspace spanned by $\left\{e_{k}\right\}$. If $z \perp Y$, then

$$
E\left(e^{i t(z, x)}\right)=C(t z)=1, \quad E(f(x))=\int_{X} f(x) P(d x),
$$

for every $t \in R^{1}$. Therefore we get

$$
P\left(L_{z}\right)=1, L_{z}=\{x:(z, x)=0\} .
$$

Since $Y=\bigcap_{z} L_{z}$, we obtain

(1) $P(Y)=1$,

because $L_{z}$ is closed and $P$ is Radon.

Now we will prove that $Y=S(P)$. For this purpose it is enough to prove that

$$
P\{x \in X:\|x-a\|<r\}>0
$$

for every $a \in Y$ and every $r>0$. Suppose to the contrary that we have $a \in Y$ and $r>0$ such that

$$
P\{x \in X:\|x-a\|<r\}=0 .
$$

Then we have

(2) $E\left(e^{-\alpha|| x-a||^{2} / 2}\right) \leqq e^{-\alpha r^{2} / 2}, \quad \alpha>0$.

On the other hand we have by (1)

$$
E\left(e^{-\alpha|| x-a \|^{2} / 2}\right)=E\left(e^{-\alpha \sum_{k}\left(x_{k}-a_{k}\right)^{2} / 2}\right), \quad x_{k}=\left(x, e_{k}\right), \quad a_{k}=\left(a, e_{k}\right) .
$$

Since

$$
E\left(e^{i \sum_{k=1}^{n} z_{k} x_{k}}\right)=\exp \left\{-\sum_{k=1}^{n} v_{i} z_{k}^{2} / 2\right\}, \quad n=1,2, \cdots,
$$

$x_{k}, k=1,2, \cdots$ are independent and each $x_{k}$ is $N\left(0, v_{k}\right)$-distributed on the probability space $(X, P)$. Thus we have 
(3) $\quad E\left(e^{-\alpha\|x-a\|^{2} / 2}\right)=\prod_{k} E\left(e^{-\alpha\left(x_{k}-a_{k}\right)^{2} / 2}\right)$

$$
=\prod_{k} \exp -\frac{\alpha a_{k}^{2}}{2\left(1-v_{k} \alpha\right)}\left(1+v_{k} \alpha\right)^{-1 / 2}
$$

Comparing (2) with (3) we have

(4) $\prod_{k} \exp \frac{a_{k}^{2} \alpha}{1+v_{k} \alpha}\left(1+v_{k} \alpha\right) \geq e^{\alpha r^{2}}$.

Writing $I_{1}$ and $I_{2}$ for the products corresponding to $k \leq N$ and $k>N$ respectively, we have

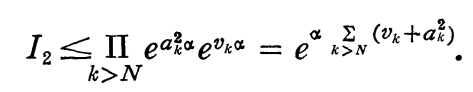

Since $\sum v_{k}$ and $\sum a_{k}^{2}$ are both finite, we have

(5) $I_{2} \leq e^{\alpha r^{2} / 2}$

for some large $N$ which is independent of $\alpha$. Fix such $N$. From (4) and

(5) we have

$$
\prod_{k=1}^{N} \exp \frac{a_{k}^{2} \alpha}{1+v_{k} \alpha}\left(1+v_{k} \alpha\right) \geq e^{\alpha r^{2} / 2}
$$

namely

$$
\prod_{k=1}^{N} \exp \frac{a_{k}^{2} \alpha}{1+v_{k} \alpha} \cdot \frac{\prod_{k=1}^{N}\left(1+v_{k} \alpha\right)}{e^{\alpha r^{2} / 2}} \geq 1
$$

Letting $\alpha \uparrow \infty$, we have

$$
\prod_{k=1}^{N} e^{a_{k}^{2} / v_{k}} \cdot 0 \geq 1
$$

which is a contradiction. This completes the proof.

Cornell University 
